\title{
TRACKING PROPERTIES OF TRAJECTORIES ON RANDOM ATTRACTING SETS
}

\author{
Tomás Caraballo and José A. Langa \\ Dpto. Ecuaciones Diferenciales y Análisis Numérico. \\ Universidad de Sevilla. Apartado de Correos 1160. \\ 41080-SEVILLA (Spain) \\ e-mails: caraball@numer.us.es, langa@numer.us.es
}

\begin{abstract}
The theory of random attracting sets highlights interesting properties of the asymptotic behaviour of some stochastic differential equations. In this paper some results on the relation between the dynamics on random attractors and stochastic inertial manifolds, and the dynamics in the associated random dynamical system are studied. In particular, some tracking properties of trajectories on random attractors and a general result on the asymptotic completeness of stochastic inertial manifolds are shown.
\end{abstract}

\section{INTRODUCTION}

One of the main concepts for the study of the asymptotic behaviour of dissipative dynamical systems is the global attractor (see Constantin et al. [4], Hale [18], Temam [26] and the references therein). This is a compact, invariant set attracting uniformly every trajectory starting in a bounded set 
of the phase space. The understanding of the dynamics on the global attractor gives us relevant information about the asymptotic behaviour of the dynamical system.

One of the most important results in the theory of global attractors claims that the fractal, and so the Hausdorff, dimension of this set is finite, even if the dynamical system is posed in an infinite-dimensional phase space. That is, although the trajectories depend on an infinite number of degrees of freedom, the finite dimensionality of the attractors seems to be describing the asymptotic behaviour of the dynamical system with a finite number of timedependent coordinates. This makes even more interesting the study of the dynamics on the global attractor, as we expect that this dynamics can be described by a system of ordinary differential equations (see Eden et al. [12], chapter 10, and Robinson [23],[24]). In fact, this is what we obtain in the theory of inertial manifolds (smooth invariant manifolds which attracts every trajectory exponentially fast, see Foias et al. [16]), that is, the dynamics on the inertial manifold, governed by a system of ordinary differential equations, is determining the asymptotic behaviour of the dynamical system. From a geometrical point of view, this fact can be seen when it is proved that every trajectory of the system can be followed arbitrarily close, as time grows to infinite, by another trajectory moving on the inertial manifold. We say in this case that the inertial manifold satisfies a tracking property (Foias et al. [17]) or that it is asymptotically complete (Robinson [22]).

In recent years, Crauel and Flandoli [7] (see also Schmalfuss [25] and Crauel et al. [6]) have introduced a concept for the attractor of some stochastic differential equations. The generalization to this situation is not trivial, as in this case the system is non autonomous and the new forcing term may have very large fluctuations which make that solutions are pushed out from any bounded ball in the phase space. However, it is possible to define in these cases a generalized concept of global attractor as a moving (in the parameters time and omega of the random term) compact set, invariant with respect to 
the shift associated to the random dynamical system (see section 2) and attracting, backwards in time, all the trajectories starting in any bounded set. This set is called random attractor and, although the convergence property is proved, roughly speaking, from $-\infty$, it is easy to show that we also have convergence in probability, forward in time, to the random attractor.

On the other hand, there are some results on the finite dimensionality (with probability one) of the random attractor (see Debussche [10], [11]), which motivates again the study of the dynamics on these random attracting sets to know in what sense it is determining the asymptotic behaviour of these stochastic differential equations. In this direction, Flandoli and Langa [14] have proved a result on determining modes for random dynamical systems which generalizes the results of Foias and Prodi [15] to the stochastic case.

In section 3, we present a generalization of a result already known in the deterministic case (see Langa and Robinson [21]).

There are also some papers extending the concept of inertial manifold to the stochastic case (see Bensoussan and Flandoli [3], Chueshov and Girya [8] or Da Prato and Debussche [9]). A stochastic inertial manifold is a random invariant Lipschitz manifold which attracts every trajectory exponentially fast. As in the deterministic case, the inertial manifold is given as the graph of some (random) Lipschitz function. In section 4, a result that shows the asymptotic completeness property of these stochastic inertial manifolds is proved. In fact, it is even more general than classical results on asymptotic completeness on inertial manifolds in the deterministic case, since it is still true for general invariant exponentially attracting sets and not necessarily smooth manifolds given as graphs of some functions. The result can be applied to the problems studied in [3] and [8]. Finally, some conclusions and possible generalizations are presented in last section. 


\section{RANDOM DYNAMICAL SYSTEMS AND ATTRACTORS}

Let $(\Omega, \mathcal{F}, P)$ be a probability space and $\left\{\theta_{t}: \Omega \rightarrow \Omega, t \in\right\}$ a family of measure preserving transformations such that $(t, \omega) \mapsto \theta_{t} \omega$ is measurable, $\theta_{0}=\mathrm{id}$, $\theta_{t+s}=\theta_{t} \theta_{s}$, for all $s, t \in$. The flow $\theta_{t}$ together with the probability space $\left(\Omega, \mathcal{F}, P,\left(\theta_{t}\right)_{t \in}\right)$ is called a (measurable) dynamical system. Furthermore, we suppose that the shift $\theta_{t}$ is ergodic.

A random dynamical system (RDS) on a Polish space $(X, \mathrm{~d})$ with Borel $\sigma$-algebra $\mathcal{B}$ over $\theta_{t}$ on $(\Omega, \mathcal{F}, P)$ is a measurable map

$$
\begin{aligned}
\varphi:^{+} \times \Omega \times X & \rightarrow X \\
(t, \omega, x) & \mapsto \varphi(t, \omega) x
\end{aligned}
$$

such that $P-$ a.s.

i) $\varphi(0, \omega)=$ id $\quad($ on $X)$

ii) $\varphi(t+s, \omega)=\varphi\left(t, \theta_{s} \omega\right) \circ \varphi(s, \omega), \forall t, s \in^{+} \quad$ (cocycle property).

A RDS is continuous or differentiable if $\varphi(t, \omega): X \rightarrow X$ is continuous or differentiable. The theory of random dynamical systems covers all systems with randomness, in particular random and stochastic difference and differential equations (see Arnold and Crauel [2]).

A random set $K(\omega)$ is said to absorb the set $B \subset X$ if $P$ - a.s. there exists $t_{B}(\omega)$ such that for all $t \geq t_{B}(\omega)$

$$
\varphi\left(t, \theta_{-t} \omega\right) B \subset K(\omega) .
$$

Finally, a random set $\mathcal{A}(\omega)$ is a random attractor associated to the $\operatorname{RDS} \varphi$ if $P-a . s$.

i) $\mathcal{A}(\omega)$ is a random compact set,

ii) $\varphi(t, \omega) \mathcal{A}(\omega)=\mathcal{A}\left(\theta_{t} \omega\right), \forall t \geq 0 \quad$ (invariance) and 
iii) for all $B \subset X$ bounded (and nonrandom)

$$
\lim _{t \rightarrow+\infty} \operatorname{dist}\left(\varphi\left(t, \theta_{-t} \omega\right) B, \mathcal{A}(\omega)\right)=0,
$$

where $\operatorname{dist}(.,$.$) denotes the Hausdorff semidistance$

$$
\operatorname{dist}(A, B)=\sup _{a \in A} \inf _{b \in B} \mathrm{~d}(a, b), \quad A, B \subset X .
$$

In this situation, we have the following theorem about existence of random attractors due to Crauel and Flandoli ([7], theorem 3.11).

THEOREM 1 Suppose there exists a compact set $D(\omega)$ absorbing every bounded nonrandom set $B \subset X$. Then, the set

$$
\mathcal{A}(\omega)=\overline{\bigcup_{B \subset X} \Lambda_{B}(\omega)}
$$

is a random attractor for $\varphi$, where the union is taken over all $B \subset X$ bounded, and $\Lambda_{B}(\omega)$ denotes the omega-limit set of $B$ which is given by

$$
\Lambda_{B}(\omega)=\bigcap_{n \geq 0} \overline{\bigcup_{t \geq n} \varphi\left(t, \theta_{-t} \omega\right) B} .
$$

Moreover, in Crauel [5] it is shown that random attractors are unique and, under the ergodicity assumption on $\theta_{t}$, there exists a compact set $K \subset X$ such that $P-$ a.s. the random attractor is the omega limit set of $K$, that is,

$$
\mathcal{A}(\omega)=\bigcap_{n \geq 0} \overline{\bigcup_{t \geq n} \varphi\left(t, \theta_{-t} \omega\right) K}
$$

\section{A TRACKING PROPERTY ON RANDOM ATTRACTORS}

In this section we shall prove a property on random attractors already known for (deterministic) global attractors (see Langa and Robinson [21]). It says, 
roughly speaking, that given a random dynamical system for which there exists a random attractor, every random trajectory can be followed arbitrarily closely by skipping from one solution to another on the random attractor.

Let us consider the following partial differential equation on the Hilbert space $H$ (norm |.|) perturbed by an additive $H$-valued white noise process on the probability space $(\Omega, \mathcal{F}, P)$ with shift $\theta_{t}$ on $\Omega$

$$
d u=-A u d t+f(u) d t+d W(t)
$$

where $A$ is a linear operator, selfadjoint, positive and with compact inverse, whose domain is $D(A)$, and $f$ is the nonlinear term. In these conditions, there exist an increasing sequence of eigenvalues of $A, \lambda_{n} \nearrow+\infty$, and the corresponding sequence of eigenfunctions $\left\{e_{n}\right\}_{n=1}^{\infty}$, which forms an orthogonal basis in $H$.

Let $\varphi:{ }^{+} \times \Omega \times H \rightarrow H$ be a random dynamical system associated to problem (1) and assume the following property of continuity with respect to initial conditions: there exists $L>0$ such that, given $s \geq 0$, it follows that, for all $u, v \in H$, for all $t \geq 0$, and $P-a . s$.

$$
\left|\varphi\left(t, \theta_{s} \omega\right) u-\varphi\left(t, \theta_{s} \omega\right) v\right| \leq e^{L t}|u-v|
$$

In addition to the precedent hypotheses, suppose the ones for the existence of a random attractor are satisfied, so that there exists a random compact set $\mathcal{A}(\omega)$, invariant, and such that, for all $B \subset H$ bounded and $P-a . s$.

$$
\lim _{t \rightarrow+\infty} \operatorname{dist}\left(\varphi\left(t, \theta_{-t} \omega\right) B, \mathcal{A}(\omega)\right)=0
$$

Since the shift $\theta_{t}$ is measure preserving, it is known (see Crauel and Flandoli [7]) that this last convergence implies convergence in probability, that is, for all $\epsilon>0$,

$$
\lim _{t \rightarrow+\infty} P\left(\operatorname{dist}\left(\varphi(t, \omega) B, \mathcal{A}\left(\theta_{t} \omega\right)\right)<\epsilon\right)=1 .
$$

¿From (2) and (3) the following result holds: 
Proposition 1 Writing a trajectory of problem (1) as $\varphi(t, \omega) u_{0}, u_{0} \in H$, and given $0<\epsilon<1,0<\delta<1$, and $T>0$, there exists a time $\tau(\epsilon, \delta, T)>0$ such that, for all $\tau \geq \tau(\epsilon, \delta, T)$, there exists $\widetilde{\Omega}$ with $P(\widetilde{\Omega})>1-\delta$ such that, if $\omega \in \widetilde{\Omega}$, there exists a point $v_{\tau} \in \mathcal{A}\left(\theta_{\tau} \omega\right)$ satisfying

$$
\left|\varphi(t+\tau, \omega) u_{0}-\varphi\left(t, \theta_{\tau} \omega\right) v_{\tau}\right|<\epsilon, \quad \text { for all } \quad 0 \leq t \leq T .
$$

Proof. Given $0<\epsilon, \delta<1, T>0$, from (3) we deduce that there exists $\tau=\tau(\epsilon, \delta, T)>0$ such that, $\forall t \geq \tau(\epsilon)$,

$$
P\left(\operatorname{dist}\left(\varphi(t, \omega) u_{0}, \mathcal{A}\left(\theta_{t} \omega\right)\right)<\epsilon e^{-L T}\right) \geq 1-\delta .
$$

In particular, for $t=\tau$, there exists $\Omega_{\tau}, P\left(\Omega_{\tau}\right) \geq 1-\delta$ such that, for all $\omega \in \Omega_{\tau}$,

$$
\operatorname{dist}\left(\varphi(\tau, \omega) u_{0}, \mathcal{A}\left(\theta_{\tau} \omega\right)\right)<\epsilon e^{-L T} .
$$

As $\mathcal{A}\left(\theta_{\tau} \omega\right)$ is compact, there exists $v_{\tau} \in \mathcal{A}\left(\theta_{\tau} \omega\right)$ such that

$$
\left|\varphi(\tau, \omega) u_{0}-v_{\tau}\right| \leq \epsilon e^{-L T}
$$

and, by (2),

$$
\left|\varphi\left(t, \theta_{\tau} \omega\right) \varphi(\tau, \omega) u_{0}-\varphi\left(t, \theta_{\tau} \omega\right) v_{\tau}\right| \leq\left|\varphi(\tau, \omega) u_{0}-v_{\tau}\right| e^{L t} \leq \epsilon,
$$

for all $t \in[0, T]$, and this gives the proposition by the cocycle property.

As a consequence of this result we obtain the following tracking property for trajectories of problem (1).

Corollary 1 Given $0<\delta<1, T>0$, and $\left\{\epsilon_{n}\right\}_{n=1}^{\infty}, \epsilon_{n}>0, \epsilon_{n} \searrow 0$, there exist a sequence of times $\left\{\tau_{n}\right\}_{n=1}^{\infty}, \tau_{n} \nearrow+\infty$,

$$
\tau_{n+1}>\tau_{n} \quad \forall n \in N, \quad \tau_{n+1}-\tau_{n} \rightarrow \infty \quad \text { as } n \rightarrow \infty,
$$

and a subset $\widetilde{\Omega} \subset \Omega$ with $P(\widetilde{\Omega})>1-\delta$, such that, for each $\omega \in \widetilde{\Omega}$, there exists $v_{\tau_{n}} \in \mathcal{A}\left(\theta_{\tau_{n}} \omega\right)$ satisfying

$$
\left|\varphi\left(t+\tau_{n}, \omega\right) u_{0}-\varphi\left(t, \theta_{\tau_{n}} \omega\right) v_{\tau_{n}}\right|<\epsilon_{n}, \quad 0 \leq t \leq n T, \quad \forall n \in
$$


Proof. Given $0<\delta<1$, we choose a decreasing sequence of $\left\{\delta_{n}\right\}_{n=1}^{\infty}$ such that

$$
\left.\sum_{n=1}^{\infty} \delta_{n}<\delta \quad \text { (for instance, } \quad \delta_{n}=\frac{\delta}{2^{n+1}}\right)
$$

Then, applying Proposition 1 for $\epsilon_{1}, \delta_{1}$ and $T$, there exists $\tau_{1}=\tau\left(\delta_{1}, \epsilon_{1}, T\right)$ such that, for all $\tau \geq \tau\left(\delta_{1}, \epsilon_{1}, T\right)$, there exists $\Omega_{\tau} \subset \Omega$ with $P\left(\Omega_{\tau}\right)>1-\delta_{1}$ satisfying that, for each $\omega \in \Omega_{\tau}$, there exists $v_{\tau} \in \mathcal{A}\left(\theta_{\tau} \omega\right)$ with

$$
\left|\varphi(t+\tau, \omega) u_{0}-\varphi\left(t, \theta_{\tau} \omega\right) v_{\tau}\right|<\epsilon_{1}, \quad \text { for all } \quad 0 \leq t \leq T
$$

Now, again by Proposition 1 for $\epsilon_{2}, \delta_{2}$ and $2 T$, we get that there exists $\tau_{2}=\tau\left(\delta_{2}, \epsilon_{2}, T\right) \geq \tau_{1}$ such that, for all $\tau \geq \tau_{2}$, there exists $\Omega_{\tau} \subset \Omega$ with $P\left(\Omega_{\tau}\right)>1-\delta_{2}$ such that, for each $\omega \in \Omega_{\tau}$, there exists $v_{\tau} \in \mathcal{A}\left(\theta_{\tau} \omega\right)$ with

$$
\left|\varphi(t+\tau, \omega) u_{0}-\varphi\left(t, \theta_{\tau} \omega\right) v_{\tau}\right|<\epsilon_{2}, \quad \text { for all } \quad 0 \leq t \leq 2 T
$$

In general, given $\epsilon_{n}, \delta_{n}$ and $n T$, there exists $\tau_{n}=\tau\left(\delta_{n}, \epsilon_{n}, T\right) \geq \tau_{n-1}$ such that, for all $\tau \geq \tau_{n}$, there exists $\Omega_{\tau} \subset \Omega$ with $P\left(\Omega_{\tau}\right)>1-\delta_{n}$ such that, for each $\omega \in \Omega_{\tau}$, there exists $v_{\tau} \in \mathcal{A}\left(\theta_{\tau} \omega\right)$ with

$$
\left|\varphi(t+\tau, \omega) u_{0}-\varphi\left(t, \theta_{\tau} \omega\right) v_{\tau}\right|<\epsilon_{n}, \quad \text { for all } \quad 0 \leq t \leq n T .
$$

Let us call $\Omega_{n} \subset \Omega$ the set with the property that, for all $\omega \in \Omega_{n}$, there exists $v_{\tau_{n}} \in \mathcal{A}\left(\theta_{\tau_{n}} \omega\right)$ satisfying

$$
\left|\varphi\left(t+\tau_{n}, \omega\right) u_{0}-\varphi\left(t, \theta_{\tau_{n}} \omega\right) v_{\tau_{n}}\right|<\epsilon_{n}, \quad 0 \leq t \leq n T
$$

We have that $P\left(\Omega_{n}\right) \geq 1-\delta_{n}$. Denoting $\widetilde{\Omega}=\cap_{n=1}^{\infty} \Omega_{n}$, it is clear from (4) that $P(\widetilde{\Omega})>1-\delta$. Now, taking $\omega \in \widetilde{\Omega}$ we have that there exists $v_{\tau_{n}} \in \mathcal{A}\left(\theta_{\tau_{n}} \omega\right)$ with

$$
\left|\varphi\left(t+\tau_{n}, \omega\right) u_{0}-\varphi\left(t, \theta_{\tau_{n}} \omega\right) v_{\tau_{n}}\right|<\epsilon_{n}, \quad 0 \leq t \leq n T
$$

for all $n \in$, and thus

$$
P\left(\left|\varphi\left(t+\tau_{n}, \omega\right) u_{0}-\varphi\left(t, \theta_{\tau_{n}} \omega\right) v_{\tau_{n}}\right|<\epsilon_{n}, \quad 0 \leq t \leq n T, \quad \forall n \in\right)>1-\delta .
$$




\subsection{Application. A reaction-diffusion equation with additive noise}

Let $D \subset^{n}$ be an open bounded set with regular boundary and

$$
f(u)=\sum_{k=0}^{2 p-1} a_{k} u^{k}, \quad a_{2 p-1}<0 .
$$

We consider the following partial differential equation of reaction-diffusion type in $D$ with an additive white noise process:

$$
\left\{\begin{aligned}
d u & =\Delta u d t+f(u) d t+\sum_{i=1}^{d} \phi^{i} d W_{t}^{i} \quad \text { in } D \\
u & =0 \text { on } \partial D \\
u(0) & =u_{0}
\end{aligned}\right.
$$

where $W_{t}^{i}: \Omega \rightarrow, t \in$, are independent one dimensional two-sided Wiener processes on a probability space $(\Omega, \mathcal{F}, P)$.

As it is well known, (5) can be expressed as a differential equation in $H=$ $L^{2}(D)$,

$$
\left\{\begin{aligned}
d u & =A u d t+F(u) d t+\sum_{i=1}^{d} \phi^{i} d W_{t}^{i} \quad \text { in } H \\
u(0) & =u_{0}
\end{aligned}\right.
$$

where $A: D(A) \subset H \rightarrow H, A u=\Delta u, F: Z \rightarrow Z^{\prime}, Z=L^{2 p}(D)$ and $Z^{\prime}=L^{(2 p)^{\prime}}(D)$, with $(2 p)^{\prime}=(2 p-1) / 2 p$, and is defined as $F(u)=f(u)$. We take $\phi^{i} \in D(A)$.

We can define a random dynamical system $\varphi(t, \omega): H \rightarrow H$ on $\left(\Omega, \mathcal{F}, P,\left(\theta_{t}\right)\right.$ $(t \in)$, with the shift $\theta_{t}$ ergodic, for which the existence of a random attractor $\mathcal{A}(\omega)$ has already been proved (for the study of the random atractor of this problem, see Crauel and Flandoli [7], Crauel et al. [6], and Debussche [10]). Due to the condition on the nonlinear term $f$, and under standard computations (see, for instance, Debussche [10]), it can be shown that $P$-a.s.

$$
\left|u\left(t, \omega ; u_{0}\right)-u\left(t, \omega ; v_{0}\right)\right| \leq e^{k t}\left|u_{0}-v_{0}\right|,
$$

so that the continuity property with respect to initial conditions (2) is satisfied and, consequently, the results in this section are true for this problem. 


\section{ASYMPTOTIC COMPLETENESS OF STOCHAS- TIC INERTIAL MANIFOLDS}

The generalization of the concept of inertial manifold to some differential equations with additive noise was introduced in Bensoussan and Flandoli [3] and Chueshov and Girya [8]. After them, Da Prato and Debussche [9] constructed a stochastic inertial manifold for a differential equation with multiplicative noise. As random attractors, a stochastic inertial manifold is a time and omega depending family of sets (manifolds), as can be seen in the following definition

DEFINITION 1 Given a random dynamical system $\varphi(t, \omega)$ on a Hilbert space $H$, associated to a stochastic differential equation, a random family of manifolds $\mathcal{M}(\omega) \subset H$ is a stochastic inertial manifold if $P-$ a.s. satisfies

i) $\mathcal{M}(\omega)$ is a Lipschitz manifold

ii) $\varphi(t, \omega) \mathcal{M}(\omega)=\mathcal{M}\left(\theta_{t} \omega\right)$ for all $t \geq 0$ and

iii) $\exists \nu>0$ such that $P-$ a.s.

$$
\lim _{t \rightarrow+\infty} e^{\nu t} \operatorname{dist}\left(\varphi(t, \omega) u_{0}, \mathcal{M}\left(\theta_{t} \omega\right)\right)=0, \quad \text { for all } u_{0} \in H .
$$

As in the deterministic case (see Foias et al. [16]), all the examples in the literature give the stochastic inertial manifold as the graph of certain random Lipschitz function $\phi_{t}(\omega): P_{m} H \rightarrow\left(I-P_{m}\right) H$ on a finite dimensional space $P_{m} H(m \in)$, that is, for $t \geq 0$ and $P-a . s$.

$$
\mathcal{M}\left(\theta_{t} \omega\right)=\left\{P_{m} u+\phi_{t}(\omega)\left(P_{m} u\right), \quad u \in H, \quad P_{m}: H \rightarrow P_{m} H\right\},
$$

where $P_{m}$ is the finite dimensional orthogonal projector on $H$ defined as $P_{m} u=\sum_{i=1}^{m}\left(u, e_{i}\right) e_{i}$, with (.,.) the inner product in $H$ and $\left\{e_{i}\right\}_{i=1}^{\infty}$ an orthonormal basis in $H$. All the examples in the literature give the Lipschitz constant $L_{\phi}$ of $\phi_{t}(\omega)$ uniform in $t \geq 0$ and $\omega \in \Omega$. We also assume (2). 
In this situation, the exponential convergence property can be written as follows: there exists $\nu>0$ such that $P-a . s$.

$$
\lim _{t \rightarrow+\infty} e^{\nu t}\left|Q_{m} \varphi(t, \omega) u_{0}-\phi_{t}(\omega)\left(P_{m} \varphi(t, \omega) u_{0}\right)\right|=0,
$$

where $Q_{m}=I-P_{m}$. Indeed, we can prove the following equivalence:

$$
\operatorname{dist}\left(\varphi(t, \omega) u_{0}, \mathcal{M}\left(\theta_{t} \omega\right)\right) \leq c_{1} e^{-\nu t}
$$

if and only if

$$
\left|Q_{m} \varphi(t, \omega) u_{0}-\phi_{t}(\omega)\left(P_{m} \varphi(t, \omega) u_{0}\right)\right| \leq c_{2} e^{-\nu t}
$$

In order to prove this, observe that

$$
\begin{aligned}
& \left|Q_{m} \varphi(t, \omega) u_{0}-\phi_{t}(\omega)\left(P_{m} \varphi(t, \omega) u_{0}\right)\right|^{2} \\
& \leq 2\left|Q_{m} \varphi(t, \omega) u_{0}-\phi_{t}(\omega)(p)\right|^{2}+2\left|\phi_{t}(\omega)(p)-\phi_{t}(\omega)\left(P_{m} \varphi(t, \omega) u_{0}\right)\right|^{2} \\
& \leq 2\left|Q_{m} \varphi(t, \omega) u_{0}-\phi_{t}(\omega)(p)\right|^{2}+2 L_{\phi}^{2}\left|p-P_{m} \varphi(t, \omega) u_{0}\right|^{2}
\end{aligned}
$$

for all $p \in P_{m} H$, so that this inequality is also true if we take the lower value moving $p \in P_{m} H$, that is,

$$
\begin{aligned}
& 2\left|Q_{m} \varphi(t, \omega) u_{0}-\phi_{t}(\omega)(p)\right|^{2}+2\left|\phi_{t}(\omega)(p)-\phi_{t}(\omega)\left(P_{m} \varphi(t, \omega) u_{0}\right)\right|^{2} \\
& \leq c \operatorname{dist}\left(\varphi(t, \omega) u_{0}, \mathcal{M}\left(\theta_{t} \omega\right)\right)^{2} \leq c c_{1}^{2} e^{-2 \nu t}
\end{aligned}
$$

A similar argument proves the other implication.

Clearly, a stochastic inertial manifold describes the asymptotic behaviour of a random dynamical system with a finite number of degrees of freedom. In particular, the results on determining modes in Flandoli and Langa [14] are straightforward in this situation, as the following lemma shows

LEMMA 1 Let $\mathcal{M}(\omega)$ be a stochastic inertial manifold given as the graph of the Lipschitz function $\phi_{t}(\omega): P_{m} H \rightarrow Q_{m} H$. If for $u_{0}, v_{0} \in H$ and $P-$ a.s.

$$
\lim _{t \rightarrow+\infty}\left|P_{m}\left(\varphi(t, \omega) u_{0}-\varphi(t, \omega) v_{0}\right)\right|=0
$$

then, $P-$ a.s.

$$
\lim _{t \rightarrow+\infty}\left|\varphi(t, \omega) u_{0}-\varphi(t, \omega) v_{0}\right|=0
$$


Proof. From the exponential convergence property (7) and the hyphotheses in the lemma we have

$$
\begin{aligned}
& \left|\varphi(t, \omega) u_{0}-\varphi(t, \omega) v_{0}\right| \\
\leq & \left|P_{m}\left(\varphi(t, \omega) u_{0}-\varphi(t, \omega) v_{0}\right)\right|+\left|Q_{m}\left(\varphi(t, \omega) u_{0}-\varphi(t, \omega) v_{0}\right)\right| \\
\leq & \left|P_{m}\left(\varphi(t, \omega) u_{0}-\varphi(t, \omega) v_{0}\right)\right|+\left|Q_{m} \varphi(t, \omega) u_{0}-\phi_{t}(\omega)\left(P_{m} \varphi(t, \omega) u_{0}\right)\right|+ \\
& +\left|\phi_{t}(\omega)\left(P_{m} \varphi(t, \omega) u_{0}\right)-\phi_{t}(\omega)\left(P_{m} \varphi(t, \omega) v_{0}\right)\right| \\
& +\left|\phi_{t}(\omega)\left(P_{m} \varphi(t, \omega) v_{0}\right)-Q_{m} \varphi(t, \omega) v_{0}\right| \\
\leq & \left(1+L_{\phi}\right)\left|P_{m}\left(\varphi(t, \omega) u_{0}-\varphi(t, \omega) v_{0}\right)\right|+ \\
+ & \left|Q_{m} \varphi(t, \omega) u_{0}-\phi_{t}(\omega)\left(P_{m} \varphi(t, \omega) u_{0}\right)\right|+\left|\phi_{t}(\omega)\left(P_{m} \varphi(t, \omega) v_{0}\right)-Q_{m} \varphi(t, \omega) v_{0}\right|,
\end{aligned}
$$

which tends to zero, and the lemma is complete.

One of the most geometrical properties about how the dynamics on an attracting set determines the asymptotic behaviour of the dynamical system is the asymptotic completeness of inertial manifolds (see Foias et al. [17], Henry [19] or Robinson [22]). In this last paper, it is proved that if an inertial manifold is flow normally hyperbolic (see definition below), then it is asymptotically complete. In this section, we shall show how this result also holds for stochastic inertial manifolds. Firstly, let us give the definition of this new concept.

Definition 2 A stochastic inertial manifold is said to be asymptotically complete if for all $u_{0} \in H$ there exists $v_{0} \in \mathcal{M}(\omega)$ such that

$$
\lim _{t \rightarrow+\infty}\left|\varphi(t, \omega) u_{0}-\varphi(t, \omega) v_{0}\right|=0
$$

Remark. Note that, for all $t \geq 0, \varphi(t, \omega) v_{0} \in \mathcal{M}\left(\theta_{t} \omega\right)$, that is, $\varphi(t, \omega) v_{0}$ describes a trajectory on the inertial manifold. Since the inertial manifold is usually defined as the graph of a (random) Lipschitz function on a finitedimensional space, the asymptotic completeness highlights geometrically the finite-dimensional asymptotic dynamics of the random dynamical system. 
Note that, as in the deterministic case (see Ladyzhenskaya [20], lemma 2.1), due to the strict invariance of the stochastic inertial manifold, we can define, for each $v_{0} \in \mathcal{M}(\omega)$, the negative semitrajectory $\varphi(-t, \omega) v_{0} \in \mathcal{M}\left(\theta_{-t} \omega\right)$, for all $t>0$. Furthermore, if, $P-$ a.s. and for all $t>0, \varphi(t, \omega)$ is one to one, the whole trajectory $(t \in)$ is unique. Indeed, a complete trajectory passing through $v_{0} \in \mathcal{M}(\omega)$ and lying on the manifold is a function $v: \rightarrow H$ satisfying

i) $v(t) \in \mathcal{M}\left(\theta_{t} \omega\right) \quad \forall t \in, \quad v(0)=v_{0}$

ii) $\varphi\left(t, \theta_{s} \omega\right) v(s)=v(t+s), \quad \forall s \in, \forall t \geq 0$.

In particular, $v(t)=\varphi(t, \omega) v_{0}$, for $t \geq 0$.

Now, given $v_{0} \in \mathcal{M}(\omega)$, we can easily construct a complete trajectory $v(t)$. For $t \geq 0$, set $v(t)=\varphi(t, \omega) v_{0}$. For $t<0$, we use the invariance of the manifold and, since $\varphi\left(1, \theta_{-1} \omega\right) \mathcal{M}\left(\theta_{-1} \omega\right)=\mathcal{M}(\omega)$, there exists $v_{-1} \in \mathcal{M}\left(\theta_{-1} \omega\right)$ such that $\varphi\left(1, \theta_{-1} \omega\right) v_{-1}=v_{0}$. Again, as $\mathcal{M}(\omega), \varphi\left(1, \theta_{-2} \omega\right) \mathcal{M}\left(\theta_{-2} \omega\right)=\mathcal{M}\left(\theta_{-1} \omega\right)$, then there exists $v_{-2} \in \mathcal{M}\left(\theta_{-2} \omega\right)$ with $\varphi\left(1, \theta_{-2} \omega\right) v_{-2}=v_{-1}$. In general, there exists $v_{-n} \in \mathcal{M}\left(\theta_{-n} \omega\right)$ with $\varphi\left(1, \theta_{-n} \omega\right) v_{-n}=v_{-(n-1)}$. Finally, we join these points in the following way: for $t<0, t \in[-n,-n+1]$,

$$
v(t)=\varphi\left(t+n, \theta_{-n} \omega\right) v_{-n}
$$

It is not difficult to show, by the cocycle property, that $v(t)$ is a complete trajectory. However, the construction shows that the negative semitrajectory could not be unique. But, if the random dynamical system is one to one, $P-a . s .$, that is, if $\varphi(t, \omega)$ is invertible, for all $t \geq 0$ and $P-$ a.s., it is clear that the choice in the above construction is unique. Thus, we can extend the random dynamical system to the whole line on the inertial manifold, and write $\varphi(t, \omega)$ for $t<0$, taking into account that $\varphi(t, \omega)=\varphi\left(-t, \theta_{t} \omega\right)^{-1}$. We can now define the following concept

Definition 3 A stochastic inertial manifold $\mathcal{M}(\omega)$ is said to be flow normally hyperbolic if there exists $\gamma<\nu$ and $D(\omega)$ such that $P-$ a.s.

$$
\left|\varphi(-t, \omega) v_{1}-\varphi(-t, \omega) v_{2}\right| \leq D(\omega) e^{\gamma t}\left|v_{1}-v_{2}\right|
$$


for all $t \geq 0$ and $v_{1}, v_{2} \in \mathcal{M}(\omega)$.

Remark. This definition can be read as follows: a stochastic inertial manifold is flow normally hyperbolic if the rate of attraction towards the inertial manifold is greater than the backward separation of two trajectories on the inertial manifold. As noted before, Robinson proved in [22] (in the deterministic case) that this is a sufficient condition for an inertial manifold to be asymptotically complete.

It is worth mentioning that, in the deterministic case, the flow normally hyperbolic property is a generalization of the classical concept of normal hyperbolicity (see Fenichel [13]) based on a linearization of the flow around a small neighbourhood of the manifold and it is used to prove results on the persistence of invariant manifolds.

We can now state the main result of this section

THEOREM 2 Suppose a stochastic inertial manifold $\mathcal{M}(\omega)$ is flow normally hyperbolic and the random variable $D\left(\theta_{t} \omega\right)$ has at most a polynomial growth in t. Then $\mathcal{M}(\omega)$ is asymptotically complete. Moreover, the rate of attraction is the same as the rate of attraction towards the inertial manifold.

Proof. Since $\mathcal{M}(\omega)$ is a stochastic inertial manifold, then $P-$ a.s. and for all $u_{0} \in H$

$$
\operatorname{dist}\left(\varphi(t, \omega) u_{0}, \mathcal{M}\left(\theta_{t} \omega\right)\right) \leq C(\omega) e^{-\nu t}, \quad \forall t \geq 0
$$

and so, for all $t \geq 0$, there exists $v_{t} \in \mathcal{M}\left(\theta_{t} \omega\right)$ such that

$$
\left|\varphi(t, \omega) u_{0}-v_{t}\right| \leq C(\omega) e^{-\nu t}, \quad \forall t \geq 0 .
$$

Define

$$
v_{\infty}(t, \omega)=\lim _{T \rightarrow+\infty} \varphi\left(t-T, \theta_{T} \omega\right) v_{T}
$$


Notice that $\varphi\left(t-T, \theta_{T} \omega\right) v_{T}$ is the value at time $t$ of the trajectory which is in $v_{T}$ at time $T$, where $v_{T}$ satisfies (9) and so

$$
v_{T} \in \mathcal{M}\left(\theta_{T} \omega\right)
$$

which implies

$$
\varphi\left(t-T, \theta_{T} \omega\right) v_{T} \in \mathcal{M}\left(\theta_{t} \omega\right), \quad \forall T \geq 0,
$$

and, as a consequence, if the limit in (10) exists,

$$
v_{\infty}(t, \omega) \in \mathcal{M}\left(\theta_{t} \omega\right), \quad \forall t \geq 0
$$

Observe that $\varphi\left(t-T, \theta_{T} \omega\right) v_{T}$ is well defined due to the strict invariance of the inertial manifold.

We are going to show that $v_{\infty}(t, \omega)$ is well defined, is a solution of the stochastic differential equation associated to the random dynamical system, and is the tracking trajectory of $\varphi(t, \omega) u_{0}$.

First of all, we want to evaluate, for $s \geq t$ and $h>0$

$$
\left|\varphi\left(t-(s+h), \theta_{s+h} \omega\right) v_{s+h}-\varphi\left(t-s, \theta_{s} \omega\right) v_{s}\right|
$$

which is, by the cocycle property,

$$
\left|\varphi\left(t-s, \theta_{s} \omega\right) \varphi\left(-h, \theta_{s+h} \omega\right) v_{s+h}-\varphi\left(t-s, \theta_{s} \omega\right) v_{s}\right|
$$

and by the flow normally hyperbolicity

$$
\begin{aligned}
& \leq D\left(\theta_{s} \omega\right) e^{-\gamma(t-s)}\left|\varphi\left(-h, \theta_{s+h} \omega\right) v_{s+h}-v_{s}\right| \\
& \leq D\left(\theta_{s} \omega\right) e^{-\gamma(t-s)}\left|\varphi\left(-h, \theta_{s+h} \omega\right) v_{s+h}-\varphi\left(-h, \theta_{s+h} \omega\right) \varphi\left(h, \theta_{s} \omega\right) v_{s}\right| \\
& \quad \leq D\left(\theta_{s} \omega\right) D\left(\theta_{s+h} \omega\right) e^{-\gamma(t-s)} e^{\gamma h}\left|v_{s+h}-\varphi\left(h, \theta_{s} \omega\right) v_{s}\right|
\end{aligned}
$$

and, by definition of $v_{s+h}, v_{s}$ and the Lipschitz property of the random dynamical system,

$$
\begin{aligned}
& \left|v_{s+h}-\varphi\left(h, \theta_{s} \omega\right) v_{s}\right| \\
& \leq\left|v_{s+h}-\varphi(s+h, \omega) u_{0}\right|+\left|\varphi\left(h, \theta_{s} \omega\right) \varphi(s, \omega) u_{0}-\varphi\left(h, \theta_{s} \omega\right) v_{s}\right| \\
& \leq C(\omega) e^{-\nu(s+h)}+e^{L h} C(\omega) e^{-\nu s}
\end{aligned}
$$


Thus, returning to (11)

$$
\begin{aligned}
& \left|\varphi\left(t-(s+h), \theta_{s+h} \omega\right) v_{s+h}-\varphi\left(t-s, \theta_{s} \omega\right) v_{s}\right| \\
& \leq D\left(\theta_{s} \omega\right) D\left(\theta_{s+h} \omega\right) e^{-\gamma(t-s)} e^{\gamma h} C(\omega) e^{-\nu s}\left(e^{-\nu h}+e^{L h}\right),
\end{aligned}
$$

and thus, for all $h \leq h_{0}$, (previously chosen),

$$
\leq D\left(\theta_{s} \omega\right) D\left(\theta_{s+h} \omega\right) e^{-\gamma t} e^{-(\nu-\gamma) s} C(\omega) K .
$$

¿From (12) we can conclude that (10) converges uniformly on bounded intervals of $[0,+\infty)$ since, for any $\tau>T$,

$$
\begin{aligned}
& \left|\varphi\left(t-\tau, \theta_{\tau} \omega\right) v_{\tau}-\varphi\left(t-T, \theta_{T} \omega\right) v_{T}\right| \\
& \leq K e^{-\gamma t} C(\omega) e^{-(\nu-\gamma) T} \sum_{n=0}^{\infty} D\left(\theta_{T+n h} \omega\right) D\left(\theta_{T+(n+1) h} \omega\right) e^{-(\nu-\gamma) n h}
\end{aligned}
$$

and, by the condition on $D(\omega)$, the series above is convergent, so that the last expression is

$$
=K_{0} e^{-\gamma t} e^{-(\nu-\gamma) T}
$$

which tends to zero uniformly for $t \in\left[0, t_{0}\right]$, for all $t_{0}>0$, as $T \rightarrow+\infty$. Therefore, the limit in (10) exists and satisfies the equation of the differential equation, since it is the uniform limit of solutions of the problem.

Moreover, it is now clear that $v_{\infty}(t, \omega)$ satisfies the tracking property for $\varphi(t, \omega) u_{0}$, since

$$
\begin{aligned}
& \left|v_{\infty}(t, \omega)-\varphi(t, \omega) u_{0}\right| \\
& \quad \leq\left|v_{\infty}(t, \omega)-v_{t}(\omega)\right|+\left|v_{t}(\omega)-\varphi(t, \omega) u_{0}\right| \\
& \quad \leq\left|\lim _{T \rightarrow \infty} \varphi\left(t-T, \theta_{T} \omega\right) v_{T}-\varphi\left(t-t, \theta_{t} \omega\right) v_{t}\right|+\left|v_{t}(\omega)-\varphi(t, \omega) u_{0}\right| \\
& \quad \leq K C(\omega) e^{-\gamma t} \sum_{n=0}^{\infty} D\left(\theta_{t+n h} \omega\right) D\left(\theta_{t+(n+1) h} \omega\right) e^{-(\nu-\gamma)(t+n h)}+C(\omega) e^{-\nu t} \\
& \quad \leq K_{0} C(\omega) e^{-\gamma t} e^{-(\nu-\gamma) t}+C(\omega) e^{-\nu t} \\
& \quad \leq C(\omega) \tilde{K}_{0} e^{-\nu t} .
\end{aligned}
$$


Remark. Note that in the proof we have not made any reference to the inertial manifold given as a graph of some Lipschitz function, so that the theorem is true for a general invariant exponentially attracting closed random set which satisfies the flow normally hyperbolic property.

\subsection{Application. A Semilinear Stochastic Differential Equation with Additive Noise}

In Bensoussan and Flandoli [3] (see also Chueshov and Girya [8]) it is proved the existence of a stochastic inertial manifold for the following differential equation with additive noise

$$
\begin{aligned}
d u(t)+A u(t) d t & =R(u(t)) d t+d W(t) \\
u(0) & =u_{0},
\end{aligned}
$$

where $A$ is a selfadjoint positive linear operator with a discrete spectrum and compact inverse, so that there exists a sequence of eigenvalues

$$
0<\lambda_{1} \leq \lambda_{2} \leq \cdots \leq \lambda_{n} \leq \cdots
$$

whose corresponding eigenvectors form an orthogonal basis of $H$. $R$ is the nonlinear term which is Lipschitz continuous with constant $L_{R}$.

Under the hypotheses on $R(u)$, it is clear that condition (2) is satisfied, and that the random variable $D(\omega)$ in (8) is independent of $\omega$. Furthermore, in [3] it is proved that under the spectral gap condition

$$
\lambda_{n+1}-\lambda_{n}>4 L_{R}
$$

there exists a stochastic inertial manifold $\mathcal{M}(\omega)$ given as the graph of some random function. In this case $\gamma=(1+M) L_{R}+\lambda_{n}$. Indeed, a bound on the separation of trajectories on $\mathcal{M}(\omega)$ is given by a bound on the separation of trajectories of the ODE

$$
d p+A p d t=P_{m} R\left(p+\phi_{t}(\omega) p\right) d t+d P_{m} W_{t}
$$


and the Lipschitz constant of $A p+P_{m} R\left(p+\phi_{t}(\omega) p\right)$ is $(1+M) L_{R}+\lambda_{n}$.

On the other part, we have that the rate of attraction $\nu=\lambda_{n+1}-L_{R}(1+$ $M$ ), where $M=L_{\phi}$, and it can be chosen to be less or equal to one (see [3]). Thus, from (13) we obtain

$$
\gamma=(1+M) L_{R}+\lambda_{n}<2 L_{R}+\lambda_{n}<\lambda_{n+1}-2 L_{R}<\lambda_{n+1}-L_{R}(1+M)=\nu,
$$

so that, as $\gamma<\nu, \mathcal{M}(\omega)$ is flow normally hyperbolic and, by theorem 2 , it is asymptotically complete.

\section{CONCLUSIONS}

Some results on the relation between the dynamics on random attracting sets and dissipative random dynamical systems have been studied.

On the other hand, it has been proved a general result for the asymptotic completeness of invariant exponentially attracting random sets, which can be successfully applied to some interesting problems in the literature for which the existence of stochastic inertial manifolds has been proved.

The application of these results to other possible examples with random attractors or stochastic inertial manifolds is an interesting problem which could lead us to more general results on tracking properties of trajectories for random attracting sets. This would improve the understanding of the asymptotic behaviour of interesting stochastic systems.

\section{ACKNOWLEDGMENTS}

The authors would like to thank Prof. James Robinson for many interesting and helpful discussions on the topic of this paper.

This work has been partially supported by D.G.I.C.Y.T. (Spain) Proyecto No. PB95-1242 


\section{References}

[1] L. Arnold, Stochastic Differential Equations. Theory and Applications. Wiley, New York (1974).

[2] L. Arnold And H. CRAuel, Random dynamical systems, Lyapunov Exponents, Proceedings, Oberwolfach, Lect. Notes in Math. 1486 (1990), 1-22.

[3] A. Bensoussan and F. Flandoli, Stochastic inertial manifold, Stochastic and Stochastic Reports, $\underline{53}$ (1995), 13-39.

[4] P. Constantin, C. Foias and R. Temam, Attractors representing turbulent flows, Mem. Amer. Math. Soc. $\underline{53}$ (1985), no 314.

[5] H.CRAUEL, Invariant measures are supported by random attractors, preprint (1996).

[6] H. Crauel, A. Debussche and F. Flandoli, Random attractors, preprint 95-27 (1995), Université de Paris-Sud.

[7] H.Crauel and F. Flandoli, Attractors for random dynamical systems, Probability Theory and Related Fields, 100 (1994), 365-393.

[8] I.D. Chueshov And T.V. Girya, Inertial manifolds for stochastic dissipative dynamical systems, Doklady of Acad. Sci. Ukraine, 7 (1994), 42-45.

[9] G. Da Prato and A. Debussche, Construction of stochastic inertial manifold using backward integration, Stochastics and Stochastics Reports, $\underline{59}(3+4)$ (1996), 305-324 
[10] A. Debussche, On the finite dimensionality of random attractors, Stoch. Anal. and Appl. 15 (4) (1997), 473-492.

[11] A. Debussche, Hausdorff dimension of a random invariant set, preprint 96-34 (1996), Université de Paris-Sud.

[12] A. Eden, C. Foias, B. Nicolaenko and R. Temam, Exponential Attractors for Dissipative Evolution Equations, RAM, Wiley, Chichester, (1994).

[13] N. Fenichel, Persistence and smoothness of invariant manifolds for flows, Indiana Univ. Math. J. $\underline{23}$ (1971), 193-226.

[14] F. Flandoli and J.A. Langa, Determining modes for dissipative random dynamical systems, preprint (1997)

[15] C. Foias and G. Prodi, Sur le comportement global des solutions non-stationnaires des equations de Navier-Stokes en dimension 2, Rend. Sem. Mat. Univ. Padova, $\underline{39}$ (1967), 1-34

[16] C. Foias, G.R. Sell and R. Temam, Inertial manifolds for nonlinear evolution equations, J. Differential Equations, 73 (1985), 309-353.

[17] C. Foias, G.R. Sell and E.S. Titi, Exponential tracking and approximation of inertial manifolds for dissipative nonlinear equations, $J$. Dyn. Diff. Eq., 1 (1989), 199-244.

[18] J.K. Hale, Asymptotic behavior of dissipative systems, Math. Surveys and Monographs no. 25, AMS, Providence 1988. 
[19] D. Henry, Geometric Theory of Semilinear Parabolic Equations, Lect. Not. in Math., $\underline{840}$ (1981), Springer-Verlag, New York.

[20] O. Ladyzhenskaya, Attractors for Semigroups and Evolution Equations. Accademia Nazionale dei Lincer series, Cambridge University Press, Cambridge (1991).

[21] J.A. Langa And J.C. Robinson, Determining asymptotic behaviour from the dynamics on attracting sets, submitted.

[22] J.C. Robinson, The asymptotic completeness of inertial manifolds, Nonlinearity, $\underline{9}$ (1996), 1325-1340.

[23] J.C. Robinson, Global attractors: topology and finite-dimensional dynamics, to appear in J.Dyn. Diff. Eq. (1997).

[24] J.C. Robinson, Some approaches to finite-dimensional behaviour in the Navier-Stokes equations, Lectures given at the Depart. de Ecuaciones Diferenciales y Análisis Numérico, University of Sevilla (1997)

[25] B. Schmalfuss, Backward cocycles and attractors of stochastic differential equations. In V. Reitmann, T. Riedrich and N Koksch, editors, International Seminar on Applied Mathematics-Nonlinear Dynamics: Attractor Approximation and Global Behaviour, pp. 185-192 (1992)

[26] R. Temam, Infinite-Dimensional Dynamical Systems in Mechanics and Physics, Springer-Verlag, New York 1988. 